\title{
A small molecule approach to degrade RAS with EGFR repression is a potential therapy for KRAS mutation-driven colorectal cancer resistance to cetuximab
}

\author{
Sang-Kyu Lee ${ }^{1,2}$, Yong-Hee Cho ${ }^{1,2}$, Pu-Hyeon Cha ${ }^{1,2}$, Jeong-Soo Yoon ${ }^{1,2}$, Eun Ji Ro ${ }^{1,2}$, Woo-Jeong Jeong ${ }^{1,2}$, Jieun Park ${ }^{1,2}$,
} Hyuntae Kim,2, Tae II Kim³, Do Sik Min ${ }^{1,4}$, Gyoonhee Han ${ }^{1,2}$ and Kang-Yell Choi ${ }^{1,2}$

\begin{abstract}
Drugs targeting the epidermal growth factor receptor (EGFR), such as cetuximab and panitumumab, have been prescribed for metastatic colorectal cancer (CRC), but patients harboring KRAS mutations are insensitive to them and do not have an alternative drug to overcome the problem. The levels of $\beta$-catenin, EGFR, and RAS, especially mutant KRAS, are increased in CRC patient tissues due to mutations of adenomatous polyposis coli (APC), which occur in $90 \%$ of human CRCs. The increases in these proteins by APC loss synergistically promote tumorigenesis. Therefore, we tested KYA1797K, a recently identified small molecule that degrades both $\beta$-catenin and Ras via GSK3 $\beta$ activation, and its capability to suppress the cetuximab resistance of KRAS-mutated CRC cells. KYA1797K suppressed the growth of tumor xenografts induced by CRC cells as well as tumor organoids derived from CRC patients having both APC and KRAS mutations. Lowering the levels of both $\beta$-catenin and RAS as well as EGFR via targeting the $\mathrm{Wnt} / \beta$-catenin pathway is a therapeutic strategy for controlling CRC and other types of cancer with aberrantly activated the Wnt/ $\beta$-catenin and EGFR-RAS pathways, including those with resistance to EGFR-targeting drugs attributed to KRAS mutations.
\end{abstract}

\section{Introduction}

The Wnt/ $\beta$-catenin and RAS/extracellular signalregulated kinase (ERK) pathways play important roles in the regulation of various cellular events, including proliferation, differentiation, and transformation. Mutations in genes in both pathways, such as adenomatous polyposis coli $(A P C)$ and $K R A S$, which occur in as many as $90 \%$ and in $40-50 \%^{1,2}$, respectively, of human colorectal cancers (CRCs), are known to be involved in the initiation and progression of CRC. Both APC and KRAS mutations synergistically promote cellular transformation and tumor

\footnotetext{
Correspondence: Kang-Yell Choi (kychoi@yonsei.ac.kr)

${ }^{1}$ Translational Research Center for Protein Function Control, Yonsei University, Seoul, Korea

${ }^{2}$ Department of Biotechnology, College of Life Science and Biotechnology, Yonsei University, Seoul, Korea

Full list of author information is available at the end of the article.

These authors contributed equally: Sang-Kyu Lee, Yong-Hee Cho.
}

growth $^{3-5}$, which is attributed to the activation of the RAS-ERK pathway via the aberrant activation of the Wnt/ $\beta$-catenin signaling caused the loss of $A P C^{6-8}$. One of the key events in the crosstalk between the two pathways is the stabilization of RAS, especially oncogenic mutant KRAS, as well as $\beta$-catenin, caused by APC loss. RAS, as well as $\beta$-catenin, is degraded via glycogen synthase kinase 3 beta (GSK3 $\beta$ )-mediated phosphorylation and subsequent poly-ubiquitination-dependent proteasomal degradation; however, the inactivation of GSK3 $\beta$ by the loss of $A P C$ stabilizes these proteins ${ }^{9,10}$.

The epidermal growth factor receptor (EGFR), a transcriptional target of the $\mathrm{Wnt} / \beta$-catenin pathway ${ }^{11,12}$, is also overexpressed in human $\mathrm{CRC}$ and plays a synergistic role with the $\mathrm{Wnt} / \beta$-catenin pathway in tumorigenesis ${ }^{13-}$ ${ }^{15}$. Therefore, therapies targeting both the $\mathrm{Wnt} / \beta$-catenin and EGFR-RAS-ERK pathways, especially those lowering 
the levels of $\beta$-catenin, RAS, and EGFR, can be ideal approaches for the treatment of CRC. Based on the identification of the mechanism of RAS destabilization by the $\mathrm{Wnt} / \beta$-catenin signaling and the large increase in both $\beta$-catenin and RAS levels in human CRC, we screened and characterized small molecules that suppress the growth of CRC cells via the destabilization of both $\beta$ catenin and RAS $^{16,17}$. KYA1797K, a small molecule derived from the chemical synthesis of an initial hit, directly interacts with the $N$-terminal regulator of $\mathrm{G}$ protein signaling (RGS) domain of axin and results in the phosphorylation and subsequent degradation of both $\beta$ catenin and RAS via the activation of GSK $3 \beta^{16}$.

The drugs targeting the EGFR-RAS-ERK pathway, such as the anti-EGFR antibody drugs including cetuximab and panitumumab, have been prescribed as therapeutic drugs for patients with cancers such as metastatic CRC. However, these drugs have limitations in their usage due to the poor efficacy or the insensitivity in patients harboring KRAS mutations ${ }^{18-20}$, and have no alternative therapy. Therefore, we tested the effects of KYA1797K on the growth and transformation of KRAS-mutated CRC cells resistant to cetuximab because KYA1797K reduces both $\beta$-catenin and RAS protein levels. KYA1797K suppresses the growth and transformation of CRC cells, reduces tumor xenograft growth and decreases the colony formation and growth of tumor organoids derived from CRC patient tissues insensitive to cetuximab due to KRAS mutations via the degradation of oncogenic KRAS and $\beta$ catenin with EGFR repression.

Together, small molecules that degrade both $\beta$ catenin and RAS via the transcriptional repression of EGFR can be a potential therapy for the treatment of CRC patients with aberrantly activated $\mathrm{Wnt} / \beta$-catenin and EGFR-RAS pathways attributed to the increase in $\beta$ catenin, EGFR, and RAS as well as to their activation by pathologically important APC, KRAS, and EGFR mutations.

\section{Materials and methods}

\section{Cell culture and reagents}

Colorectal cancer (CRC) cells, including HCT15, RKO, SW480, and DLD1, were purchased from the American Type Culture Collection (ATCC, Manassas, VA, USA). Isogenic human CRC cells, DLD-1 cells harboring wildtype (WT), or mutant (MT) KRAS (D-WT and D-MT, respectively) ${ }^{21}$ were provided by B. Vogelstein (John Hopkins University School of Medicine, Baltimore, MD, USA). DiFi cells were provided by Won-Ki Kang (Division of Hematology-Oncology at Samsung Medical Center, Seoul, Republic of Korea). All the cell lines were authenticated using short tandem repeat profiling (Cosmogenetech, Seoul, Republic of Korea). RKO cells were cultured in DMEM (Gibco Life Technologies, Grand
Island, NY, USA) supplemented with $10 \%$ fetal bovine serum (FBS; Gibco Life Technologies). HCT15, DLD1, SW480, DiFi, D-WT, and D-MT cells were maintained in RPMI 1640 medium (Gibco Life Technologies) supplemented with $10 \%$ FBS. For the establishment of stable cell lines, D-WT cells were transfected with Myc-KRAS-WTpcDNA3.1, Myc-KRAS-G12V-pcDNA3.1, or emptypcDNA3.1 plasmid, and were selected in a medium containing G-418 (Sigma-Aldrich; St. Louis, MO, USA). For the establishment of the APC knockout (KO) cell lines, RKO cells were transfected with the LentiVRISPRv2 vector containing the $A P C$ gene guide sequence and were selected in a medium containing puromycin (SigmaAldrich, St. Louis, MO, USA). Lipofectamine (Invitrogen, Waltham, MA, USA) and Lipofector-EZ (AptaBio, Gyeonggi, Republic of Korea) were used for plasmid transfection according to the manufacturer's instructions. KYA1797K was dissolved in dimethyl sulfoxide (DMSO) (Sigma-Aldrich) for the in vitro studies. Cetuximab (C225, Erbitux) was obtained from Merck (Darmstadt, Germany).

\section{Plasmids}

The plasmids Myc-APC-pCMV were provided by Eric R. Fearon (University of Michigan, USA). The constructs Myc-WT-KRAS-pcDNA3.1 and Myc-G12V-KRASpcDNA3.1 were previously generated ${ }^{9}$. To construct the APC knockout vector using the CRISPR-CAS system, we designed and synthesized the guide oligos targeting the APC gene (upstream of the NGG PAM sequence) using the following sequences: forward, $5^{\prime}$ CACCGTCGCTCTTCATGGATTTTTA-3'; and reverse, 5'-AAACTAAAAATCCATGAAGAGCGAC-3' (Bioneer, Daejeon, Republic of Korea). Two oligos were annealed and ligated into the BsmB1-digested lentiCRISPRv2 vector (Addgene, Cambridge, MA, USA), and transformed into the JM109 bacterial strain. All the constructs and mutations were confirmed by nucleotide sequencing analyses (Cosmogenetech, Daejeon, Republic of Korea).

\section{Human CRC tissue microarray (TMA) analyses}

The normal, polyp/adenoma, and adenocarcinoma samples were analyzed using CRC TMA (Biomax, Inc., Rockville, MD, USA). Immunohistochemical analyses were performed with anti-EGFR, anti- $\beta$-catenin, and anti-pan-RAS antibodies. The TMA slides were visualized by microscopy (Eclipse 80i; Nikon, Melville, NY, USA). For quantitative analyses, the $\mathrm{H}$-score of each stained sample was determined by IHC profiler software (Image J) using the following scale: $\mathrm{H}$-score $=3 \times$, highly positive population, $+2 \times$, positive population, $+1 \times$, weak positive population, and $+0 \times$, negative population. 


\section{Animal study}

All animal experiments were performed in accordance with the Korean Food and Drug Administration guidelines. The protocols were reviewed and approved by the Institutional Animal Care and Use Committee (IACUC) of Yonsei University. C57BL/6J-Apc $c^{\text {min/+ }}\left(A p c^{\text {min/+ }}\right)$ and B6.129S-Kras ${ }^{\text {tm } 3 t y j}$ (Kras ${ }^{G 12 D}$ LA2) mice were obtained from the Jackson Laboratory. To generate $A p c^{\mathrm{min} /+} / \mathrm{Kras}^{G 12 D} L A 2$ mice for the tumor organoid experiment, $A p c^{\min /+}$ mice were crossed with $\operatorname{Kras}^{G 12 D} L A 2$ mice. For the xenograft study, athymic $n u / n u$ mice were injected subcutaneously in the dorsal flank with D-MT $\left(1 \times 10^{7}\right.$ cells/mouse $)$ in $200 \mu \mathrm{L}$ of PBS: Matrigel ${ }^{\circ}$ (1:1; BD Biosciences, San Jose, CA, USA). When the mean tumor size was between $100-200 \mathrm{~mm}^{3}$, the mice were randomly divided into four groups: vehicle, cetuximab, KYA1797K, or co-treatment with KYA1797K and cetuximab. The tumors were measured using Vernier calipers every 3 days, and the volumes were then calculated according to the following formula: $\pi / 6 \times$ length $\times$ width $\times$ height. Twenty-one days after the drug treatment, the mice were killed, and the tumors were excised and fixed in 4\% paraformaldehyde (PFA; Wako, Richmond, VA, USA) or snap frozen in liquid nitrogen for further analyses.

\section{Tumor organoid experiments}

For the human tumor organoid experiments, the use of human tissue was reviewed and approved by the Institutional Review Board of Severance Hospital, Yonsei University College of Medicine. The biopsied tumor samples from CRC patients were cut into $3-5 \mathrm{~mm}$ pieces, washed with ice-cold PBS three times, and incubated with collagenase II $(2 \mathrm{mg} / \mathrm{mL})$, hyaluronidase $(20 \mu \mathrm{g} / \mathrm{mL})$, and Ly27632 $(10 \mu \mathrm{M})$ for $1 \mathrm{~h}$ at $37^{\circ} \mathrm{C}$. Small intestinal tumors from $\mathrm{Apc}^{\mathrm{Min} /+} / \mathrm{Kras}^{G 12 D} \mathrm{LA2}$ mice were isolated and washed with ice-cold PBS, and single cells isolated from the tumors were collected using $0.25 \%$ trypsin containing $10 \mathrm{mM} \mathrm{Ly} 27632$ and $100 \mu \mathrm{g} / \mathrm{mL}$ Primocin ${ }^{\mathrm{m}}$ for $30 \mathrm{~min}$. After incubation, $1 \times \mathrm{B} 27$ was added, and the mixture was filtered through $100 \mu \mathrm{m}$ and $40 \mu \mathrm{m}$ cell strainers (BD Biosciences, San Jose, CA, USA) to collect single cells. The cells were mixed with growth factor-reduced Matrigel ${ }^{\circ}$ (BD Bioscience). After the gel solidified, N2 medium containing $10 \%$ R-spondin- $1 \mathrm{CM}, 100 \mu \mathrm{g} / \mathrm{mL}$ noggin, 1.25 $\mathrm{mM} N$-acetyl cysteine, $10 \mathrm{mM}$ nicotinamide, $50 \mathrm{ng} / \mathrm{mL}$ EGF, $10 \mathrm{nM}$ gastrin, $500 \mathrm{nM}$ A83-01, $3 \mu \mathrm{M}$ SB202190, and $10 \mathrm{nM}$ prostaglandin E2 was added. The growth medium was refreshed every 2 days, and the cells were passaged by mechanical disruption every 10-14 days at a 1:5 split ratio. The measurements of tumor organoid growth were performed by a CellTiter-Glo assay (Promega, Madison, WI, USA) according to the manufacturer's instructions. Luminescence was measured using a FLUOstar Optima instrument (BMG Labtech, Cary, NC, USA).

\section{Immunoblotting}

Immunoblot analysis was performed as previously described ${ }^{16}$. Immunoblotting was performed with the following antibodies: anti-pan-RAS (sc-4), anti- $\beta$-catenin (sc-7199), anti-ERK (sc-514302), anti-APC (sc-896), antiPCNA (sc-56), anti-KRAS (sc-30), and anti-Myc (sc-40) purchased from Santa Cruz Biotechnology; anti-p-ERK (9101) and anti- $\alpha$-tubulin (3873) obtained from Cell Signaling Technology; and anti-EGFR (ab52894) and anti-pEGFR (ab5644) purchased from Abcam (Cambridge, United Kingdom).

\section{GTP-RAS pull-down assays}

For the detection of GTP-RAS (active RAS), the GSTRaf1 RAS-binding domain (RBD) fusion protein was purified from cell lysates of transformed BL21 cells using glutathione-agarose beads and then resuspended in GSTFISH buffer [10\% glycerol, $50 \mathrm{mM}$ Tris (pH 7.4), $100 \mathrm{mM}$ $\mathrm{NaCl}, 1 \% \mathrm{NP}-40$, and $2 \mathrm{mM} \mathrm{MgCl}_{2}$ ] with a $50 \%$ bead slurry. Purified GST-Raf1 RBD proteins were incubated with the WCL of D-WT or D-MT cells treated under the indicated conditions, and the beads were washed with GST-FISH buffer at least five times. The pull-down samples were subjected to immunoblot analyses.

\section{Cell proliferation assays}

For the cell proliferation assays, CRC cells were plated at a density of $2-5 \times 10^{3}$ cells/well in a 96-well plate. The cells were then treated under the indicated conditions for 96 h. After incubation, 3-(4,5-dimethylthiazol-2-yl)-2-5diphenyltetrazolium bromide (MTT; Amresco, Solon, $\mathrm{OH}$, USA) reagent was added to each well at a concentration of $0.25 \mathrm{mg} / \mathrm{mL}$ for $2 \mathrm{~h}$ at $37^{\circ} \mathrm{C}$. Insoluble purple formazan was obtained by removing the medium and extracting it with DMSO. The absorbance at $590 \mathrm{~nm}$ was monitored with the FLUOstar Optima microplate reader (BMG Labtech).

\section{Colony formation assays}

CRC cells were seeded in 12-well plates (250-600 cells/ well). The cells were treated under the indicated conditions for 7-16 days with changes of the medium every 4 days. At the end of the experiment, the cells were washed, fixed, and stained with $0.5 \%$ crystal violet in $20 \%$ ethanol for $2 \mathrm{~h}$, and subsequently washed three times with distilled water.

\section{Reverse transcription and quantitative real-time PCR}

The total RNA was isolated using TRIzol $^{\circ}$ reagent (Invitrogen, Waltham, MA, USA) according to the manufacturer's instructions. The total RNA $(2 \mu \mathrm{g})$ was reverse transcribed using 200 units of M-MLV reverse transcriptase (Invitrogen) in a $20 \mu \mathrm{L}$ reaction mixture at $42{ }^{\circ} \mathrm{C}$ for $1 \mathrm{~h}$. For quantitative real-time PCR analyses, the 
resulting cDNA $(1 \mu \mathrm{L})$ was amplified in $10 \mu \mathrm{L}$ of Rotorgene SYBR ${ }^{\circ}$ green (Qiagen, Hilden, Germany). The comparative cycle threshold $\left(C_{\mathrm{T}}\right)$ method was used, and $\beta$-actin served as an endogenous control. The primers are listed in the supplementary table S1.

\section{Immunohistochemistry}

Immunohistochemistry (IHC) was performed as previously described $^{17}$. For peroxidase IHC analysis, sections were incubated with the primary antibody overnight at $4^{\circ}$ $C$, followed by incubation with biotinylated anti-mouse (Vector Laboratories, Burlingame, CA, USA) or biotinylated anti-rabbit (Vector Laboratories) secondary antibodies at RT for $1 \mathrm{~h}$. The samples were incubated in the avidin-biotin complex solution (Vector Laboratories), they were then stained with 3, 3' diaminobenzidine (DAB; Vector Laboratories) for $5 \mathrm{~min}$ and counterstained with Mayer's hematoxylin (Muto, Tokyo, Japan). The signals were analyzed using a bright field microscope (Nikon TE2000U). The fluorescence signal was visualized using a confocal microscope (LSM510; Carl Zeiss, Oberkochen, Germany) at excitation wavelengths of $488 \mathrm{~nm}$ (Alexa Fluor 488), $543 \mathrm{~nm}$ (Alexa Fluor ${ }^{\circ} 555$ ), and $405 \mathrm{~nm}$ (DAPI). At least three fields of view per section were analyzed. The IHC images were quantified from the representing images using an IHC profiler in the ImageJ software.

\section{Immunocytochemistry}

Cells were seeded on collagen-coated $(500 \mu \mathrm{g} / \mathrm{mL})$ coverslips. The cells were then fixed with $5 \%$ formalin for 30 min, permeabilized with $0.1 \%$ Triton X-100 for $20 \mathrm{~min}$, and preblocked with PBS containing $5 \%$ bovine serum albumin (BSA; Affymetrix, Santa Clara, CA, USA) and 1\% normal goat serum (NGS; Vector Laboratories) for $1 \mathrm{~h}$. The cells were then incubated with the indicated primary antibody overnight at $4{ }^{\circ} \mathrm{C}$, followed by Alexa Fluor 488 (Life Technologies, Carlsbad, CA, USA) or Alexa Fluor 555 (Life Technologies) secondary antibodies for $4 \mathrm{~h}$ at $4^{\circ}$ $\mathrm{C}$ and counterstained with DAPI for $10 \mathrm{~min}$ at room temperature. After incubation, the cells were mounted in Gel/Mount media (Biomeda Corporation, Foster City, CA, USA). The fluorescence signal was visualized using a confocal microscope (LSM510; Carl Zeiss) at excitation wavelengths of $488 \mathrm{~nm}$ (Alexa Fluor ${ }^{\circ} 488$ ), $543 \mathrm{~nm}$ (Alexa Fluor 555), and $405 \mathrm{~nm}$ (DAPI).

\section{Mutational analyses of human CRC patient samples}

To analyze the mutational status of both APC (chr5, NM_000038.5) and KRAS (chr12, NM_033360.3) in the CRC patient samples, total genomic DNA was isolated from the tumor organoids and analyzed by whole exome sequencing (Macrogen).

\section{Statistical analysis}

All the data are represented as the means \pm standard deviations of at least three independent experiments. The statistical significance was assessed using the Student's $t$ test. The significance was denoted as " $P<0.05, "{ }^{* \prime \prime} P<0.01$, and ${ }^{* * * *} P<0.001$.

\section{Results}

The levels of $\beta$-catenin, RAS, and EGFR are increased in CRC patient tissues

Given that both $\beta$-catenin and RAS are stabilized in CRC patient tissues mostly having APC mutations and that EGFR is a transcriptional target of the Wnt $/ \beta$-catenin pathway, we tested whether expression levels of $\beta$-catenin, RAS, and EGFR are correlated in CRC patient tissues. The levels of the EGFR were higher along with $\beta$-catenin and pan-RAS, especially in the polyp/adenoma and adenocarcinoma regions of the CRC patient tissues, compare with paired normal tissues (Fig. 1a, b). Similar correlated increases of the proteins were observed in $A P C$ - or CTNNB1 (encoding $\beta$-catenin)-mutated CRC cell lines (Fig. 1c). In addition, two independent CRC cohorts from the oncomine database indicated that the mRNA level of EGFR in the CRC tissues including adenoma, carcinoma, or adenocarcinoma is higher than that in the normal colon tissues (Supplementary Figure S1). These results are correlated with the protein level pattern of EGFR in the CRC patient tissue microarrays (TMAs). To further confirm these increases in the $\beta$-catenin, pan-RAS, and EGFR levels by pathologically meaningful $A P C$ loss in CRC, we generated RKO cells with the knockout (KO) of APC. The levels of $\beta$-catenin, pan-RAS, and EGFR were simultaneously increased (Fig. 1d), and the mRNA levels of the EGFR and CCND1 (cyclin D1) genes, both wellknown Wnt/ $\beta$-catenin signaling response genes, were increased in the $A P C-K O$ RKO cells (Fig. 1e). Furthermore, the growth and transforming abilities of the $A P C$ KO RKO cells were significantly elevated compared with those of the RKO cells (Fig. 1f, g). In addition, all of the $A P C$ knockout effects were rescued by the overexpression of APC in $A P C-K O$ RKO cells (Fig. 1h, i).

The Wnt/ $\beta$-catenin pathway-dependent co-regulation of EGFR and RAS was also confirmed by the increased levels of these proteins induced by recombinant Wnt3a treatment in both HEK293 and RKO cells harboring wildtype (WT) APC and CTNNB1 (Supplementary Figure S2a) and by the decreased levels of these proteins induced by the knockdown of $\beta$-catenin in both HCT15 and DLD1 cells harboring APC mutations (Supplementary Figure S2b). The mRNA levels of EGFR and CCND1 were increased and decreased by Wnt3a treatment and $\beta$ catenin knockdown, respectively (Supplementary Figure S2c and d). Therefore, EGFR and $\beta$-catenin/RAS are 


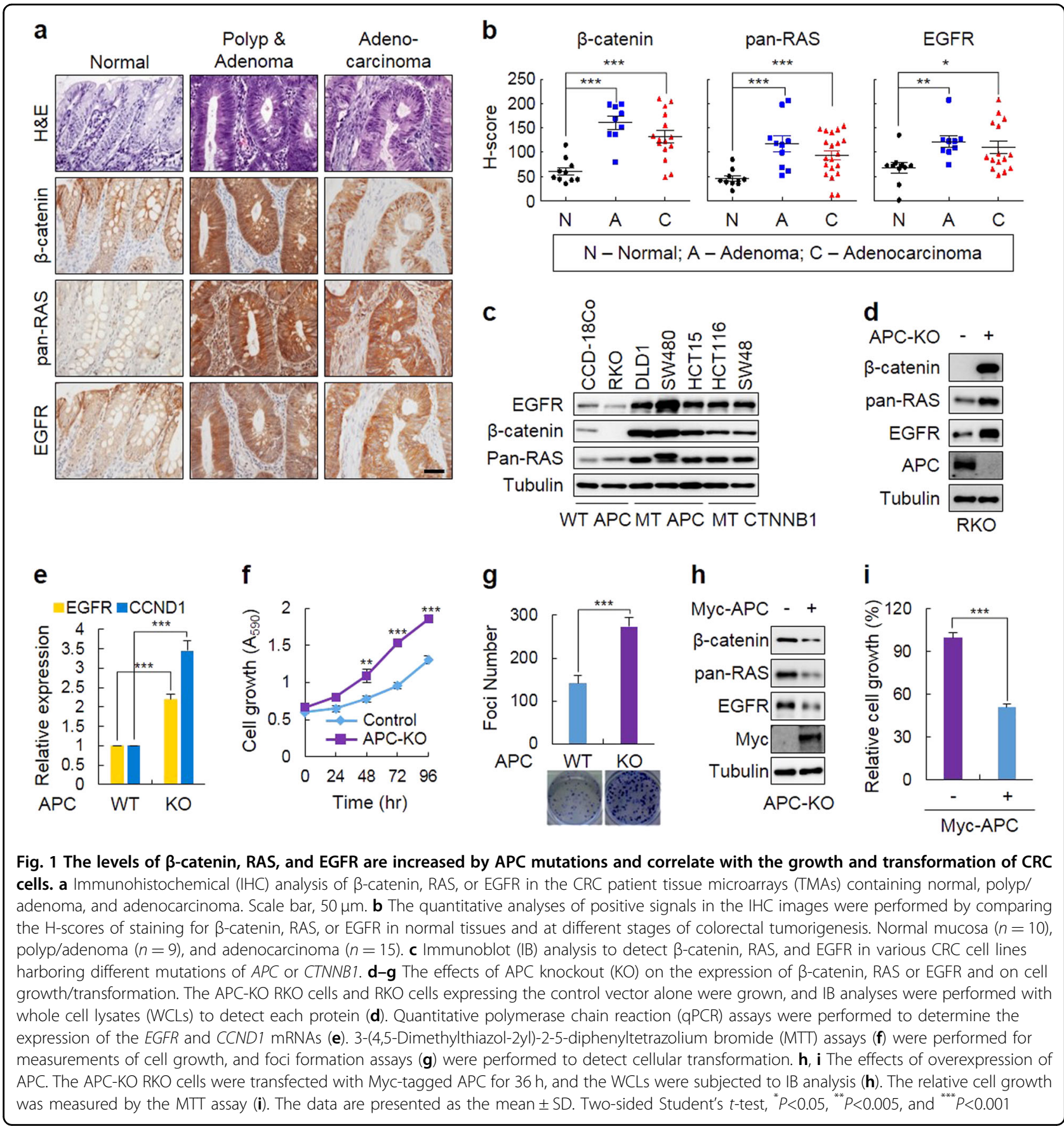

increased by the loss of APC in CRC cells at both the transcriptional and protein levels.

\section{The stabilization as well as the mutation of RAS contributes to the cetuximab resistance of CRC cells}

Although drugs targeting the EGFR, such as cetuximab, have been used for the treatment of CRC, their efficacies were limited because the drugs were ineffective for the treatment of patients having KRAS mutations ${ }^{18-20}$.
Similarly, the growth of KRAS-mutated D-MT, HCT15, and SW480 cells was not significantly inhibited, while the growth of DiFi and D-WT cells harboring WT KRAS was effectively inhibited by cetuximab (Fig. 2a).

The resistance to cetuximab in CRC cells with KRAS mutations was further investigated by using D-WT cells, a DLD-1-derived cell line that harbors only WT $K R A S^{21}$. Although $90 \%$ of the growth of D-WT cells was inhibited by cetuximab treatment for $96 \mathrm{~h}$, only $10 \%$ of the growth 


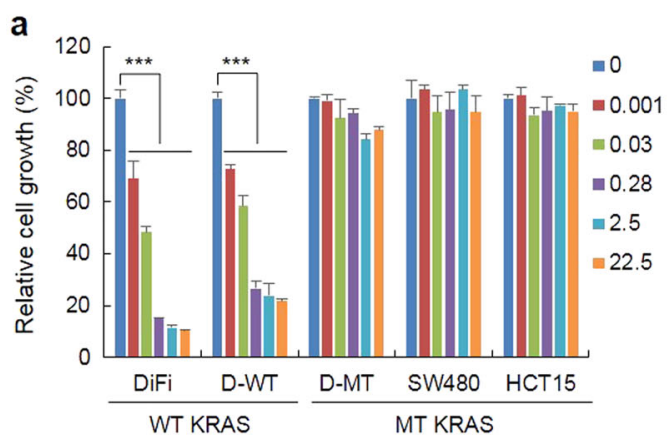

C

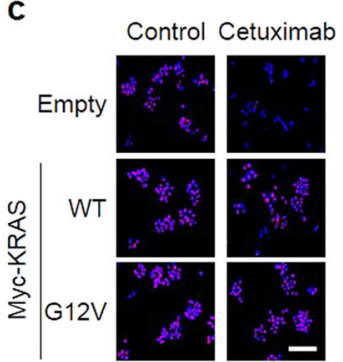

d

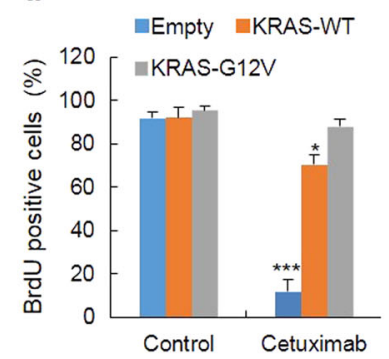

b

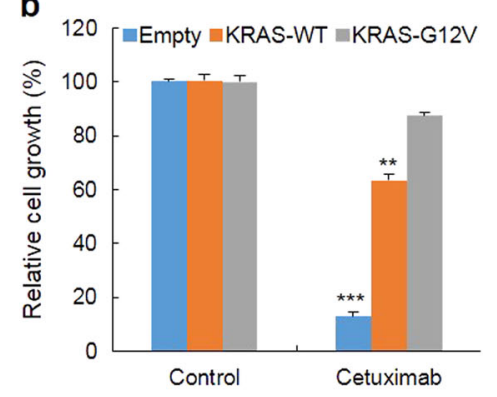

e

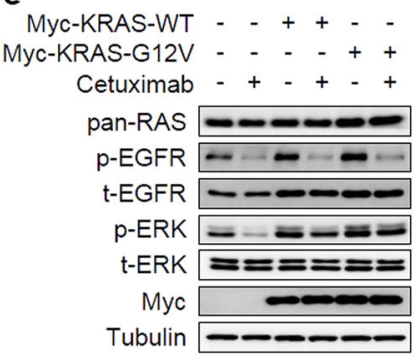

Fig. 2 The overexpression of WT KRAS, as well as the mutant form, contributes to the resistance to cetuximab. a MTT assays were performed to determine the effects of cetuximab on the growth of various CRC cells treated with the indicated doses of cetuximab for $96 \mathrm{~h}$. $\mathbf{b}$-e The effects of the overexpression of KRAS WT or oncogenic mutant G12V on cell growth. The D-WT cells stably expressing Myc-KRAS-WT, -G12V, or empty vector were treated with $5 \mu \mathrm{g} / \mathrm{mL}$ cetuximab for $96 \mathrm{~h}$. MTT (b) and bromodeoxyuridine (BrdU) incorporation (c, d) assays were performed to measure cell proliferation. Scale bar, $100 \mu \mathrm{m}$. The WCLs were subjected to IB analyses (e). The data are presented as the mean \pm SD $(n=3)$. Two-sided Student's $t-$ test, ${ }^{*} P<0.05,{ }^{* *} P<0.005$, and ${ }^{* * *} P<0.001$

was inhibited in cells overexpressing MT (G12V)-KRAS (Fig. 2b). Interestingly, cetuximab resistance was also observed for the overexpression of WT KRAS, although the degree of resistance was somewhat lower (65\%) (Fig. 2b). These results were similar to the inhibitory patterns of cell proliferation measured by the bromodeoxyuridine (BrdU) incorporation assays (Fig. 2c, d). The patterns of antiproliferation induced by KRAS overexpression correlated with the ERK activity in the cells treated with cetuximab (Fig. 2e). Overall, the resistance to the EGFR inhibitor cetuximab affecting CRC cell growth was due to the increase in the level as well as the mutation of KRAS.

KYA1797K, a small molecule that degrades both $\beta$-catenin and RAS, overcomes cetuximab resistance in CRC

Because the increase in $\beta$-catenin, RAS, and EGFR levels in CRC contributes to synergistically promote cell growth and transformation, we tested the effects of KYA1797K on the growth of CRC cells, especially cells resistant to cetuximab, due to KRAS mutations. KYA1797K dosedependently inhibited the growth of various CRC cells regardless of their KRAS mutational status (Fig. 3a), while the growth of CCD18-Co, normal colon cell, was mildly affected by KYA1797K treatment (Supplementary
Figure S3). The effectiveness of KYA1797K but not cetuximab on the inhibition of the growth and transformation of cells harboring KRAS mutations was confirmed by comparing WT- and MT-KRAS CRC cells (Fig. 3b, c; Supplementary Figure S4a and b). Co-treatment with cetuximab and KYA1797K cumulatively inhibited the growth of D-WT cells but not D-MT cells (Fig. 3b). The differential effects of co-treatment with KYA1797K and cetuximab on the growth of CRC cells, which are dependent upon the mutational status of KRAS, were also observed in a dose-dependent manner in WT KRAS DWT and DiFi cells but not in the KRAS-mutated D-MT and SW480 cells (Fig. 3d).

The inhibition of ERK activity, as well as GTP-RAS activity, by treatment with cetuximab or KYA1797K alone or in combination in CRC cells with different KRAS mutations was correlated with cell growth and transformation (Fig. 3e and Supplementary Figure S4c). The levels of $\beta$-catenin, RAS, and EGFR were reduced by KYA1797K but not by cetuximab although the p-EGFR levels were reduced by both cetuximab and KYA1797K treatment in WT and MT KRAS cells. Consistently, the EGFR mRNA levels were reduced without a change in the $\beta$-catenin mRNA levels by KYA1797K but not by cetuximab alone (Supplementary Figure S5). Overall, it was shown that 


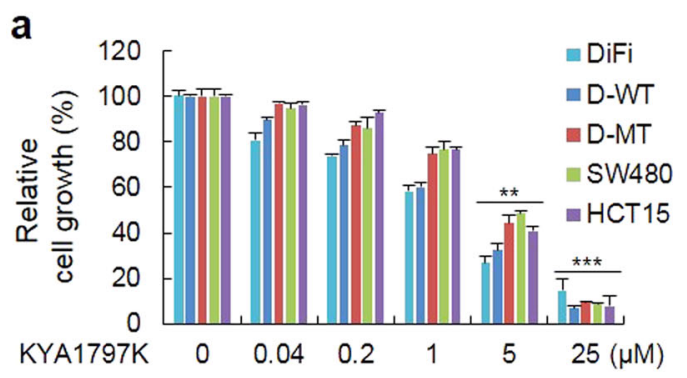

C

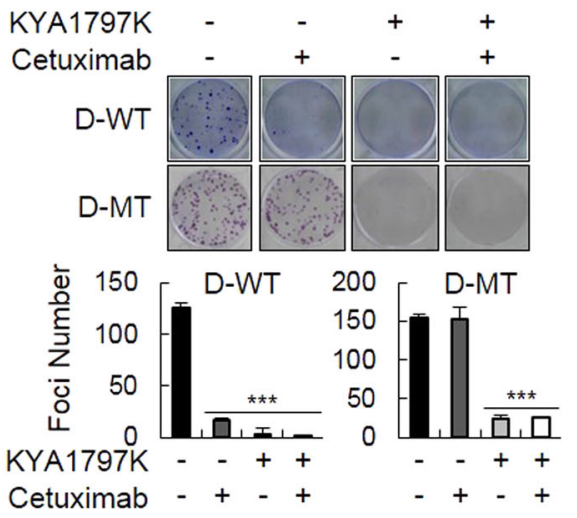

\section{e}

KYA1797K - - + + - - + +

Cetuximab - + - + + - +

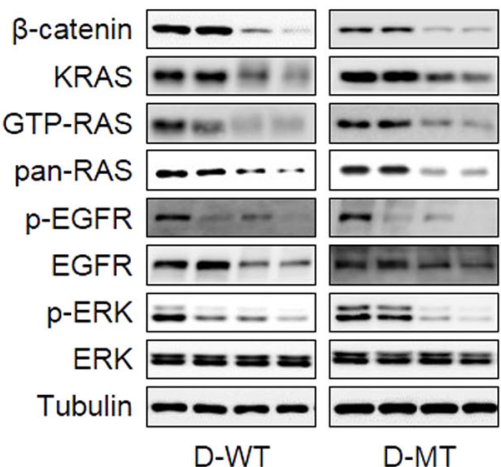

b

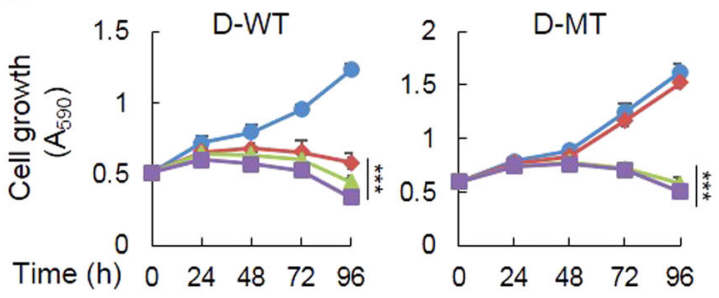

- Vehicle
- KYA1797K Cetuximab
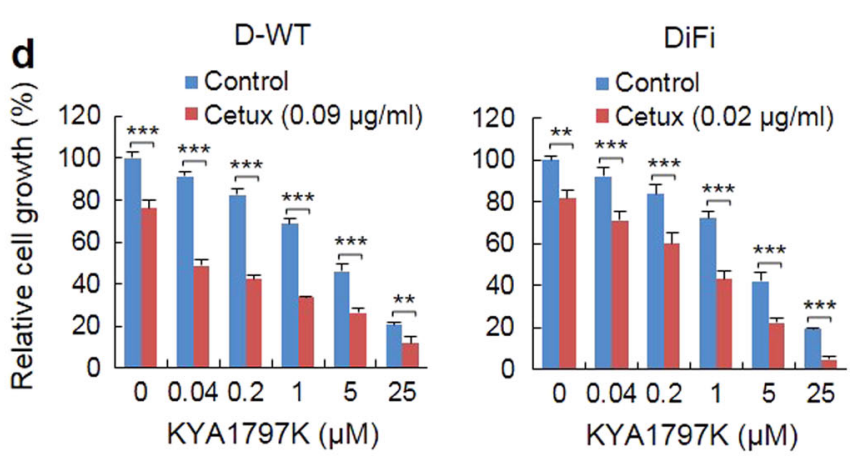

D-MT

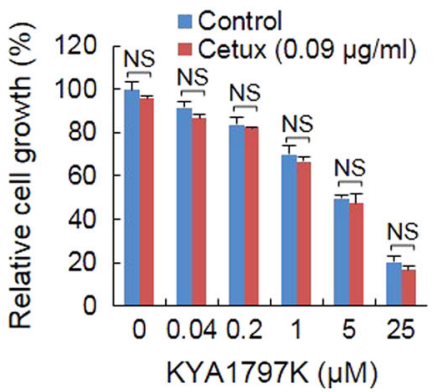

SW480

Control

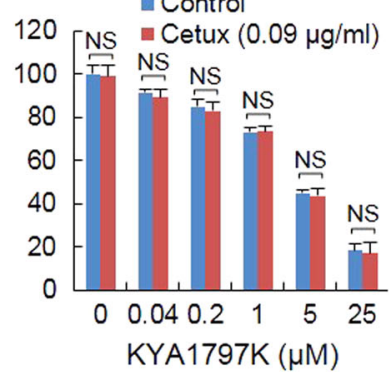

Fig. 3 KYA1797K overcomes the resistance to cetuximab attributed to KRAS mutations on the growth and transformation of CRC cells. a MTT assays were performed to determine the effects of KYA1797K on the growth of various CRC cells treated with the indicated doses of KYA1797K

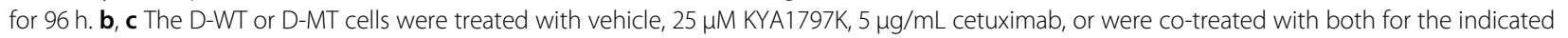
time periods. The growth and transformation of the D-WT or D-MT cells were monitored by MTT (b) and foci formation (c) assays, respectively. $\mathbf{d}$ MTT assays were performed to determine the combined effect of KYA1797K and cetuximab in D-WT, DiFi, D-MT, and SW480 cells co-treated with the indicated doses of KYA1797K and cetuximab for $96 \mathrm{~h}$. e IB analysis of D-WT or D-MT cells treated under the indicated conditions for $24 \mathrm{~h}$. GlutathioneS-transferase (GST)-Raf-1 RAS-binding domain (RBD) pull-down assays were performed to detect active RAS (GTP-RAS). The data are presented as the mean \pm SD $(n=3)$. Two-sided Student's $t$-test, ${ }^{* *} P<0.005$, and ${ }^{* * *} P<0.001$. NS not significant

KYA1797K degrades both $\beta$-catenin and RAS proteins with the transcriptional repression of EGFR. Treatment with KYA1797K overcame the ineffectiveness of cetuximab for inhibiting the colony formation ability and growth of CRC cells harboring KRAS mutations.
KYA1797K overcomes the resistance to cetuximab attributed to KRAS mutations in tumor xenografts

The effectiveness of KYA1797K in the cetuximabresistant CRC cells harboring KRAS mutations was also tested in vivo using a xenograft mouse model that 

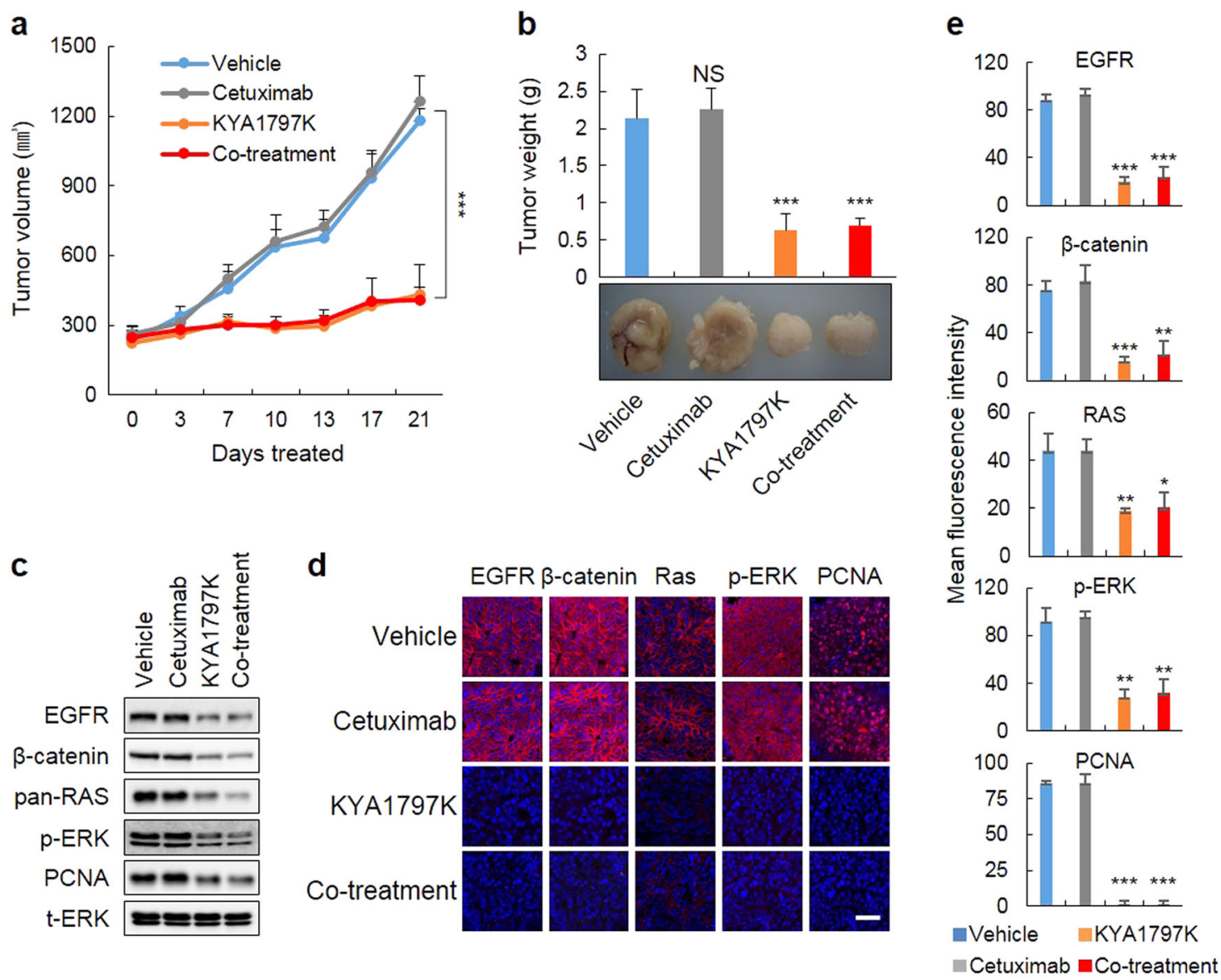

Fig. 4 KYA1797K overcomes the resistance to cetuximab attributed to KRAS mutations on tumor xenograft growth. a D-MT cells were subcutaneously injected into nude mice with the subsequent intraperitoneal (i.p.) injection of vehicle, $20 \mathrm{mg} / \mathrm{kg} \mathrm{KYA1797K,1} \mathrm{mg/mouse} \mathrm{cetuximab,}$ or a combination of KYA1797K ( $20 \mathrm{mg} / \mathrm{kg})$ and cetuximab $(1 \mathrm{mg} / \mathrm{mouse})$ for 21 days. The tumor volumes were measured every 3 days. b The measurement of tumor weights (b, upper panel) and the visualization of tumor images (b, lower panel) were performed at the time of killing. $\mathbf{c}$ WCLs prepared from the tumor tissues were subjected to IB analysis. $\mathbf{d} I \mathrm{HC}$ analysis of the tissue sections incubated with the indicated antibodies and then counterstained with 4',6-diamidino-2-phenylindole (DAPI). Scale bar, $50 \mu \mathrm{m}$. e The levels of the proteins were measured by the mean fluorescence intensity. The data are presented as the mean \pm SD $(n=5)$. Two-sided Student's $t$-test, ${ }^{*} P<0.05,{ }^{* *} P<0.005$, and ${ }^{* * *} P<0.001$. NS not significant

produced tumors after the implantation of D-MT cells harboring both $A P C$ and KRAS mutations. Mice intraperitoneally (i.p.) injected with cetuximab did not show an effect in tumor growth, whereas mice treated with KYA1797K alone or co-treated with KYA1797K and cetuximab showed a significantly reduced tumor volume and weight (Fig. 4a, b). In agreement with the results of the in vitro studies, treatment with KYA1797K alone or in combination with cetuximab but not cetuximab alone reduced ERK activity and reduced $\beta$-catenin, RAS, EGFR, and proliferating cell nuclear antigen (PCNA) protein levels, as shown by the immunoblots (Fig. 4c), immunohistochemical analyses, and subsequent quantification (Fig. 4d, e). Together, these results indicate that
KYA1797K efficiently suppressed the growth of tumors and overcame the insensitivity to cetuximab in the KRASmutated cells via degradation of the KRAS protein.

KYA1797K suppresses the growth of cetuximab-resistant tumor organoids derived from $\mathrm{Apc}^{\mathrm{Min} /+} / \mathrm{Kras}^{\mathrm{G} 12 \mathrm{D}} \mathrm{LA2}$ mice or CRC patients harboring mutant KRAS

To further validate the effectiveness of KYA1797K but not cetuximab, we adapted a tumor organoid culture system. Similar to the results of the tumor xenograft studies, both the formation and growth of the tumor organoids from the small intestine of $A p c^{\mathrm{Min} /+} / \mathrm{Kras}^{\mathrm{G} 12-}$ ${ }^{D} L A 2$ mice having both $A p c$ and Kras mutations were specifically suppressed by KYA1797K (Fig. 5a, b). 

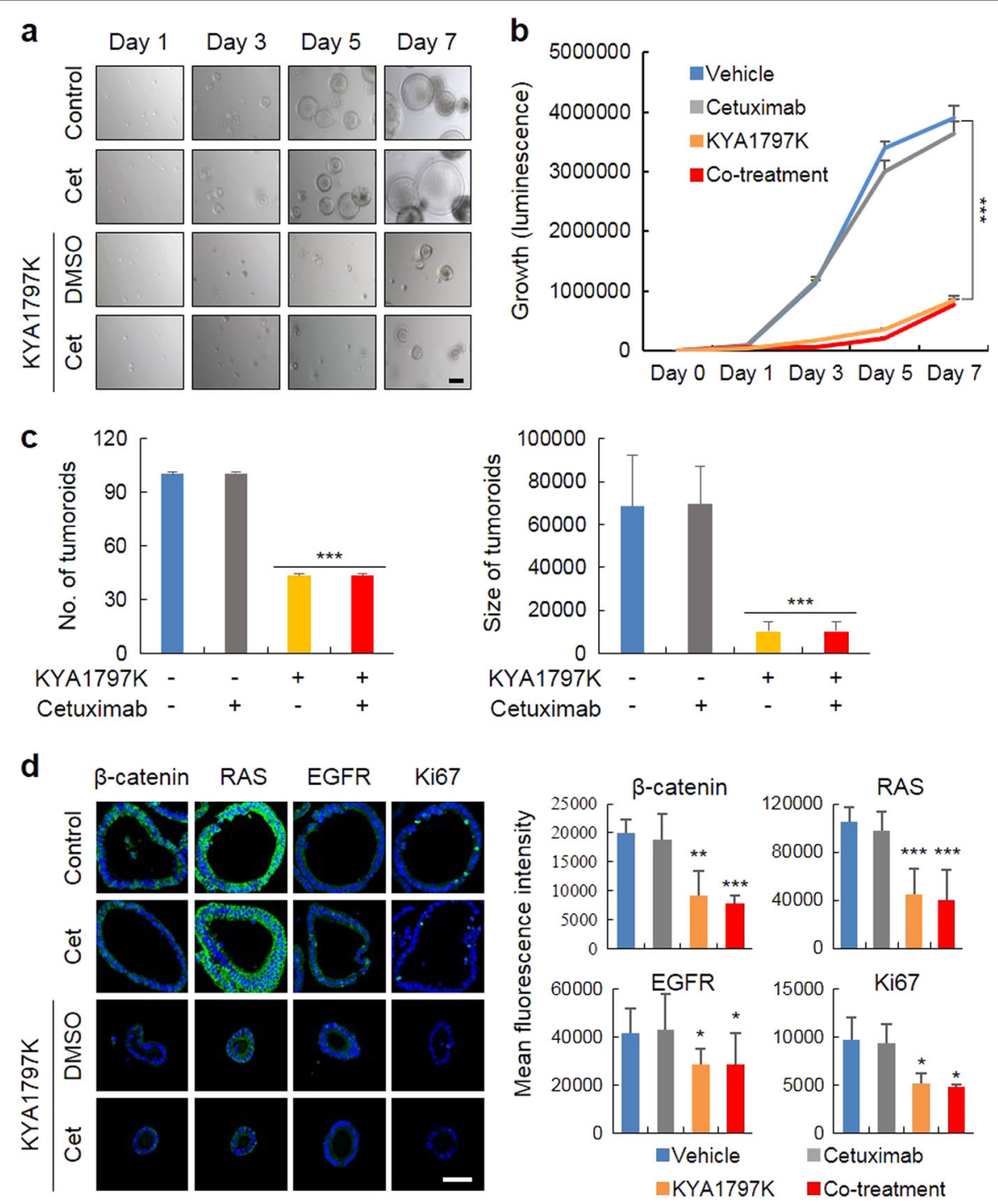

Fig. 5 The effectiveness of KYA1797K on the formation and growth of tumor organoids from the small intestine of $A p c^{\mathrm{Min} /+} / \mathrm{Kras}^{\mathrm{G12D}} \mathrm{LA2}$ mice. The tumor organoids from the small intestine of $A p C^{\mathrm{Min} /+} / \mathrm{Kras}^{G 12 \mathrm{D}} \mathrm{LA2}$ mice were cultured as described in the materials and methods section. The tumor organoids were cultured and treated under the indicated conditions for 7 days. $\mathbf{a}, \mathbf{b}$ The images of the tumor organoids were visualized (a, scale bar, $200 \mu \mathrm{m}$ ), and the growth of the tumor organoids was measured by a cell titer assay (b). c The numbers (left) and sizes (right) of the tumor organoids were quantified. $\mathbf{d}$ Immunocytochemical (ICC) analysis of $\beta$-catenin, pan-RAS, EGFR, and Ki67 in the tumor organoids treated under the indicated conditions for 7 days. The tumor organoids were immobilized, immunostained with each antibody, and counterstained with DAPI (d, left panel). Scale bar, $50 \mu \mathrm{m}$. The levels of these proteins were measured by the mean fluorescence intensity (d, right panel). The data are presented as the mean \pm SD $(n=3)$. Two-sided Student's $t$-test, ${ }^{*} P<0.05,{ }^{* *} P<0.005$, and ${ }^{* * *} P<0.001$

Therefore, both the numbers and sizes of the tumor organoids were significantly reduced by treatment with KYA1797K, while no change was observed for treatment with cetuximab (Fig. 5c). The levels of $\beta$-catenin, RAS, EGFR, and Ki-67 were similarly reduced by KYA1797K but not by cetuximab (Fig. $5 \mathrm{~d}$ ). Consistent with the results using the KRAS-mutated CRC cell lines, the combined effects of KYA1797K and cetuximab on the growth inhibition were not observed in the tumor organoids derived from the small intestinal tumor cells of $\mathrm{Apc}^{\mathrm{Min} /+}$ / Kras $^{G 12 D} L A 2$ mice.

To test the potential application of KYA1797K in humans, we established tumor organoids derived from CRC patient tissues harboring both APC (truncated form, Arg216"stop) and KRAS (Gly12Ser) mutations, which were identified by whole exome sequencing (Fig. 6a). The 
a

\begin{tabular}{c|c|c|c}
\hline \multicolumn{4}{c}{ Mutational Status of APC and KRAS } \\
\hline $\begin{array}{c}\text { APC (chr5, NM_000038.5) } \\
\begin{array}{c}\text { Nucleotide } \\
\text { change }\end{array}\end{array}$ & KRAS (chr12, NM_033360.3) \\
\hline C.646C $>\mathrm{T}$ & $\begin{array}{c}\text { p.Arg216* } \\
\text { (stop) }\end{array}$ & $\begin{array}{l}\text { Nucleotide } \\
\text { change }\end{array}$ & Consequence \\
\hline
\end{tabular}

d

Vehicle Cetuximab $\frac{\text { KYA1797K }}{$\cline { 3 - 3 } \text {$DMSO Cetuximab }}$
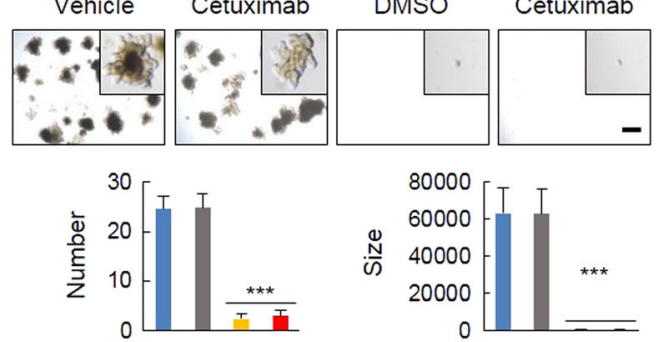

KYA1797K - - + +

Cetuximab - + - +

e

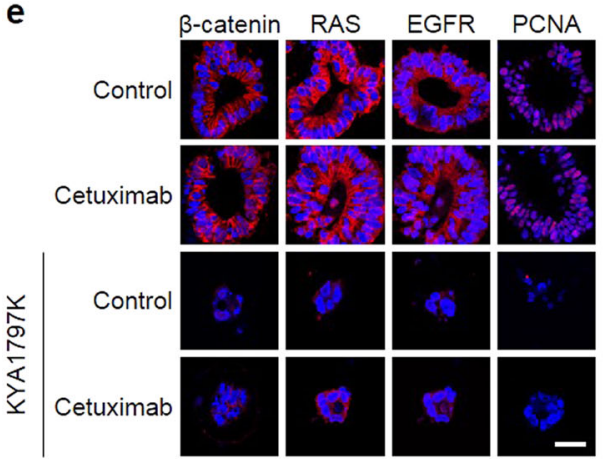

b

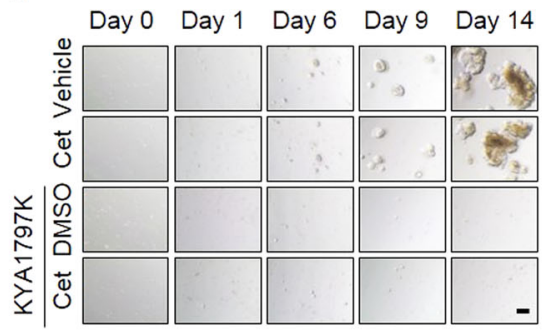

C

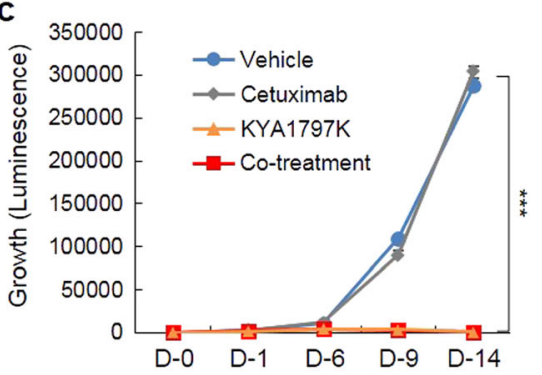

f

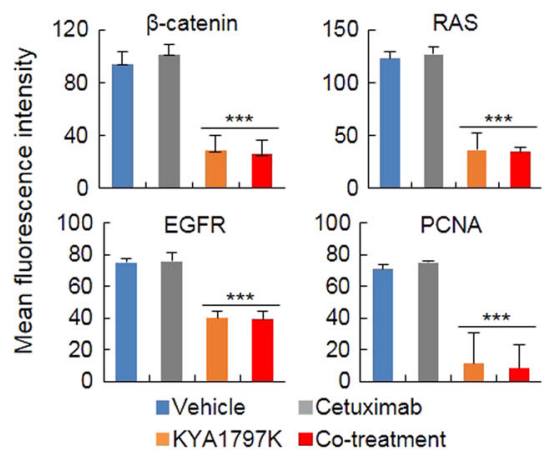

Fig. 6 KYA1797K effectively suppresses the formation and growth of tumor organoids derived from CRC patients having both APC and KRAS mutations. a The tumor organoids derived from the CRC patient tissue were cultured as described in the materials and methods section and analyzed by whole exome sequencing to determine the mutational status of the genes of interest, including KRAS and APC. $\mathbf{b}, \mathbf{c}$ The tumor organoids were treated under the indicated conditions for 14 days. The tumor organoids were visualized (b, scale bar, $200 \mu m$ ), and the growth of the tumor organoids was measured by a cell titer assay (c). $\mathbf{d}$ Images of the tumor organoids treated with the indicated conditions for 21 days were captured at low magnification (upper panel, scale bar, $1 \mathrm{~mm}$ ), and high-magnification images were visualized in the boxed region (inset). The number and size of the tumor organoids were quantified (d, lower panel). e ICC analysis of $\beta$-catenin, pan-RAS, EGFR, or PCNA in the tumor organoids cultured under the indicated conditions for 14 days. The tumor organoids were immobilized and then immunostained with antibodies. Scale bar, $50 \mu \mathrm{m}$. $\mathbf{f}$ The levels of these proteins were measured by the mean fluorescence intensity. The data are presented as the mean $\pm \operatorname{SD}(n=3)$. Two-sided Student's $t$-test, ${ }^{* * *} P$ $<0.001$

human tumor organoids were well-developed with gland formation in a time-dependent manner, which recapitulates tumor heterogeneity containing different cell populations $^{22}$. However, KYA1797K effectively inhibited gland formation in tumor organoids (Supplementary Figure S6). Although cetuximab was not effective, the formation and growth of tumor organoids were mostly abolished by KYA1797K (Fig. 6b, c). Therefore, the number and size of the tumor organoids were significantly reduced by treatment with KYA1797K, while no change was shown for treatment with cetuximab (Fig. 6d). The inhibitory effects of KYA1797K on the tumor organoids correlated with the reduction in the $\beta$-catenin, pan-RAS, EGFR, and PCNA levels (Fig. 6e, f). Overall, KYA1797K effectively suppressed the formation and growth of tumor-derived organoids from CRC patients and overcame the insensitivity to cetuximab attributed to KRAS mutations by reducing the protein levels via degradation.

\section{Discussion}

Cancer development is a dynamic and multistep process involving multiple genetic changes and molecular 
alterations ${ }^{23,24}$. In CRC, mutations of genes in the Wnt $/ \beta$ catenin and RAS/ERK pathways, such as APC and KRAS, synergistically promote transformation ${ }^{9,25}$. Aberrant activation of the $\mathrm{Wnt} / \beta$-catenin and RAS/ERK pathways by the co-stabilization of $\beta$-catenin and RAS as well as by genetic mutations in these pathway components contribute to colorectal tumorigenesis. EGFR is also overexpressed in human CRC and plays a role in synergistic tumorigenesis ${ }^{14}$.

Therefore, the inhibition of both the Wnt/ $\beta$-catenin and EGFR-RAS-ERK pathways, especially by reducing the levels of the proteins elevated in CRC, can be an ideal approach for the treatment of human CRC. However, a clinically applicable drug that inhibits both the Wnt/ $\mathrm{B}$ catenin and EGFR-RAS-ERK pathways or even the Wnt/ $\beta$-catenin pathway alone is not available. Small molecules such as KYA1797K reduce the levels of $\beta$-catenin, RAS, and EGFR and are potential drug candidates to resolve the problem of resistance to EGFR-targeting therapy attributed to KRAS mutations. The effectiveness of KYA1797K on the inhibition of cetuximab-resistant KRAS-mutated CRC cells is shown by both in vitro and in vivo studies using the mouse xenograft tumor model generated from CRC cells harboring KRAS mutations. The effectiveness and potential application of this compound in patients are demonstrated by the reduction in these proteins and the suppression of growth in tumor organoids derived from CRC patient tissues harboring both APC and KRAS mutations.

The small molecule drugs that reduce the levels of $\beta$ catenin, RAS, and EGFR are highly effective for the treatment of CRC because of the elevated protein levels caused by the loss of $A P C$, which is observed in $~ 90 \%$ of human CRCs. The approach to controlling CRC by the specific degradation of the $\beta$-catenin and RAS proteins can be further supported by a recent study suggesting that the degradation of specific oncogenic proteins is an ideal approach for cancer therapy ${ }^{26}$. Our approach to controlling RAS, especially oncogenic mutant KRAS, via its degradation provides an alternative approach for the development of a clinically applicable drug targeting the undruggable $\operatorname{RAS}^{27-30}$. Although $\beta$-catenin- and RASdestabilizing compounds induced the degradation of HRAS and NRAS, these could be beneficial for the treatment of cancer, because the overexpression of WTHRAS or -NRAS is known to play roles in the promotion of MT-KRAS-driven tumorigenesis ${ }^{31}$. The importance of the additional KRAS mutations in colorectal tumorigenesis with $A P C$ mutations is shown by the role of MT KRAS in the strong secondary activation of Wnt/ $\beta$-catenin signaling, which involves cancer stem cell activation by a positive feedback loop via the activation of ERK after the initial activation of the pathway caused by the loss of $A P C^{25}$.
In summary, we show that the levels of $\beta$-catenin, RAS, and EGFR are upregulated in a correlated manner by the loss of $A P C$, and increases in these proteins critically promote colorectal tumorigenesis. Therefore, small molecules that degrade both $\beta$-catenin and RAS with the reduction of EGFR transcription can be potential drug candidates for the treatment of CRC, including CRCs that are resistant to the EGFR-targeting therapies because of KRAS mutations.

\section{Acknowledgements}

We thank Drs. B. Vogelstein and K.W. Kinzler for providing the DLD-1 isogenic cells. This study was supported by National Research Foundation of Korea (NRF) grants funded by the Korean government (MSIP) (Grants 2016R1A5A1004694, 2015R1A2A1 A05001873). Grant: This study was supported by National Research of Korea (NRF) grants funded by the Korean government (MSIP) (Grants: 2016R1A5A1004694, 2015R1A2A1A05001873)

\section{Author details}

${ }^{1}$ Translational Research Center for Protein Function Control, Yonsei University, Seoul, Korea. ${ }^{2}$ Department of Biotechnology, College of Life Science and Biotechnology, Yonsei University, Seoul, Korea. ${ }^{3}$ Department of Internal Medicine and Institute of Gastroenterology, College of Medicine, Yonsei University, Seoul, Korea. ${ }^{4}$ Department of Molecular Biology, College of Natural Science, Pusan National University, Pusan, Korea

Conflict of interest

The authors declare that they have no conflict of interest.

\section{Publisher's note}

Springer Nature remains neutral with regard to jurisdictional claims in published maps and institutional affiliations.

Supplementary information accompanies this paper at https://doi.org/ 10.1038/s12276-018-0182-2.

Received: 22 June 2018 Revised: 9 August 2018 Accepted: 13 August 2018. Published online: 20 November 2018

\section{References}

1. Downward, J. Targeting RAS signalling pathways in cancer therapy. Nat. Rev. Cancer 3, 11-22 (2003).

2. Kinzler, K. W. \& Vogelstein, B. Lessons from hereditary colorectal cancer. Cell 87, 159-170 (1996).

3. D'Abaco, G. M., Whitehead, R. H. \& Burgess, A. W. Synergy between Apc min and an activated ras mutation is sufficient to induce colon carcinomas. Mol. Cell. Biol. 16, 884-891 (1996).

4. Horst, D. et al. Differential WNT activity in colorectal cancer confers limited tumorigenic potential and is regulated by MAPK signaling. Cancer Res. $\mathbf{7 2}$, 1547-1556 (2012)

5. Sansom, O. J. et al. Loss of Apc allows phenotypic manifestation of the transforming properties of an endogenous K-ras oncogene in vivo. Proc. Natl Acad. Sci. USA 103, 14122-14127 (2006).

6. Yun, M.-S., Kim, S.-E., Jeon, S. H., Lee, J.S. \& Choi, K-Y. Both ERK and Wnt/betacatenin pathways are involved in Wnt3a-induced proliferation. J. Cell Sci. 118 (Pt 2), 313-322 (2005).

7. Park, K-S. et al. APC inhibits ERK pathway activation and cellular proliferation induced by RAS. J. Cell Sci. 119(Pt 5), 819-827 (2006).

8. Jeon, S. H. et al. Axin inhibits extracellular signal-regulated kinase pathway by Ras degradation via beta-catenin. J. Biol. Chem. 282, 14482-14492 (2007).

9. Jeong, W. J. et al. Ras stabilization through aberrant activation of Wnt/betacatenin signaling promotes intestinal tumorigenesis. Sci. Signal. 5, ra30 (2012).

10. Kim, S. E. et al. H-Ras is degraded by Wnt/beta-catenin signaling via beta-TrCPmediated polyubiquitylation. J. Cell Sci. 122(Pt 6), 842-848 (2009). 
11. Tan, X. et al. Epidermal growth factor receptor: a novel target of the Wnt/betacatenin pathway in liver. Gastroenterology 129, 285-302 (2005).

12. Guturi, K. K. N. et al. Mechanism of $\beta$-catenin-mediated transcriptional regulation of epidermal growth factor receptor expression in glycogen synthase kinase $3 \beta$-inactivated prostate cancer cells. J. Biol. Chem. 287, 18287-18296 (2012).

13. Gross, M. et al. Cellular growth response to epidermal growth factor in colon carcinoma cells with an amplified epidermal growth factor receptor derived from a familial adenomatous polyposis patient. Cancer Res. 51, 1452-1459 (1991).

14. Hu, T. \& Li, C. Convergence between Wnt- $\beta$-catenin and EGFR signaling in cancer. Mol. Cancer 9, 1 (2010).

15. Radinsky, R. et al. Level and function of epidermal growth factor receptor predict the metastatic potential of human colon carcinoma cells. Clin. Cancer Res. 1, 19-31 (1995)

16. Cha, P. H. et al. Small-molecule binding of the axin RGS domain promotes beta-catenin and Ras degradation. Nat. Chem. Biol. 12, 593-600 (2016).

17. Cho, Y. H. et al. KY1022, a small molecule destabilizing Ras via targeting the Wnt/beta-catenin pathway, inhibits development of metastatic colorectal cancer. Oncotarget 7, 81727-81740 (2016).

18. Amado, R. G. et al. Wild-type KRAS is required for panitumumab efficacy in patients with metastatic colorectal cancer. J. Clin. Oncol. 26, 1626-1634 (2008).

19. Karapetis, C. S. et al. K-ras mutations and benefit from cetuximab in advanced colorectal cancer. N. Engl. J. Med. 359, 1757-1765 (2008),
20. Lievre, A. et al. KRAS mutations as an independent prognostic factor in patients with advanced colorectal cancer treated with cetuximab. J. Clin. Oncol. 26, 374-379 (2008).

21. Yun, J. et al. Glucose deprivation contributes to the development of KRAS pathway mutations in tumor cells. Science 325, 1555-1559 (2009).

22. Shroyer, N. F. Tumor organoids fill the niche. Cell. Stem. Cell. 18, 686-687 (2016).

23. Fearon, E. R. \& Vogelstein, B. A genetic model for colorectal tumorigenesis. Cell 61, 759-767 (1990)

24. Loeb, L. A., Loeb, K. R. \& Anderson, J. P. Multiple mutations and cancer. Proc Natl. Acad. Sci. USA 100, 776-781 (2003).

25. Moon, B.-S. et al. Role of oncogenic K-Ras in cancer stem cell activation by aberrant Wnt/B-catenin signaling. J. Natl. Cancer Inst. 106, djt373 (2014).

26. Crunkhorn, S. Anticancer drugs: Selectively targeting proteins for degradation. Nat. Rev. Drug Discov. 14, 459 (2015).

27. Downward, J. RAS synthetic lethal screens revisited: still seeking the elusive prize? Clin. Cancer Res. 15, 1802-1809 (2015).

28. Simanshu, D. K., Nissley, D. V. \& McCormick, F. RAS proteins and their regulators in human disease. Cell 170, 17-33 (2017).

29. Papke, B. \& Der, C. J. Drugging RAS: know the enemy. Science 355, 1158-1163 (2017).

30. Dang, C. V., Reddy, E. P., Shokat, K. M. \& Soucek, L. Drugging the'undruggable'cancer targets. Nat. Rev. Cancer 17, 502-508 (2017).

31. Grabocka, E. et al. Wild-type $\mathrm{H}$ - and N-Ras promote mutant K-Ras-driven tumorigenesis by modulating the DNA damage response. Cancer Cell $\mathbf{2 5}$, 243-256 (2014) 